\title{
Low-frequency, Repetitive Transcranial Magnetic Stimulation for the Treatment of Patients with Posttraumatic Stress Disorder: a Double-blind, Sham-controlled Study
}

\author{
Dong-Hyun Nam, Chi-Un Pae, Jeong-Ho Chae \\ Department of Psychiatry, The Catholic University of Korea College of Medicine, Seoul, Korea
}

\begin{abstract}
Objective: Several studies have suggested that repetitive transcranial magnetic stimulation (rTMS) of the right prefrontal cortex may be useful in the treatment of posttraumatic stress disorder (PTSD). The aim of this study was to compare the effect of rTMS on the right prefrontal cortex with that of sham stimulation among patients with PTSD.

Methods: In total, 18 patients with PTSD were randomly assigned to the $1-\mathrm{Hz}$ low-frequency $\mathrm{rTMS}$ group or the sham group for 3 weeks. Primary efficacy measures were the Clinician-Administered PTSD Scale (CAPS) and its subscales, assessed at baseline and at 2, 4, and 8 weeks.

Results: All CAPS scores improved significantly over the study period. We found significant differences in the re-experiencing scores ( $F=7.47, p=0.004)$ and total scores ( $F=6.45, p=0.008)$ on the CAPS. The CAPS avoidance scores showed a trend toward significance $(F=2.74, p=0.055)$, but no significant differences in the CAPS hyperarousal scores were observed.

Conclusion: The present study showed low-frequency rTMS to be an effective and tolerable option for the treatment of PTSD. Trials using variable indices of rTMS to the right prefrontal cortex and explorations of the differences in the effects on specific symptom clusters may be promising avenues of research regarding the use of rTMS for PTSD.
\end{abstract}

KEY WORDS: Repetitive transcranial magnetic stimulation; Posttraumatic stress disorder; Clinician-Administered Posttraumatic Stress Disorder Scale.

\section{INTRODUCTION}

Repetitive transcranial magnetic stimulation (rTMS) is a recently introduced non-invasive tool that has been recommended as a possible treatment for various psychiatric disorders. The feasibility of the application of rTMS to the treatment of psychiatric disorders became a subject of interest after it was reported that rTMS can alter the cortical excitability of the brain. ${ }^{1)}$ It is evident that rTMS has effects on various brain functions, such as motor function, memory, concentration, language, and emotion. Some reports have suggested that rTMS induced significant changes in a monoamine receptor in the cerebral cortex and had a substantial and rapid effect on the monoamine

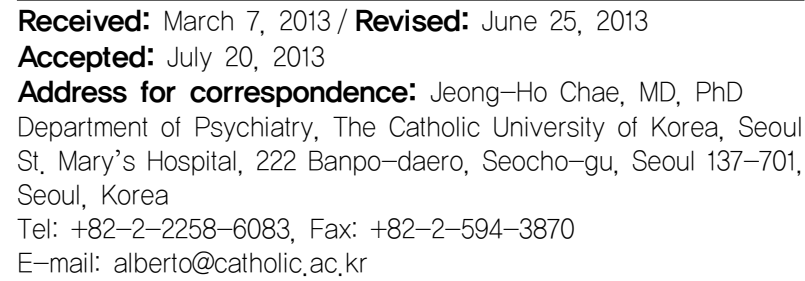

neurotransmitters system. ${ }^{2,3)}$ It also has modulatory effects on thyroid stimulatory hormone and cortisol, ${ }^{4)}$ suggesting that this technique could be applicable to the alterations in the neuroendocrine system in mood disorders. rTMS has been used most in the treatment of depressive disorders, ${ }^{5)}$ and its application to psychiatric disorders has recently been extended to obsessive-compulsive disorder, ${ }^{6)}$ tic disorder, ${ }^{7)}$ and even to major psychosis. ${ }^{8,9)}$

Considering the pathophysiology of posttraumatic stress disorder (PTSD), rTMS may be applicable to the treatment of PTSD. Previous findings from a number of functional neuroimaging studies and the predominance of hyperarousal symptom in PTSD suggest that altered brain excitability could be a major factor in the pathogenesis of PTSD. ${ }^{10-13)}$ Moreover, a recent neurophysiological study reported that alterations in intracortical inhibition could be implicated in the pathogenesis of PTSD. Measurement of motor-evoked potentials revealed that the lack of intracortical inhibition was strictly correlated with the presence of PTSD symptoms and that functional or structural injury to highly excitable interneurons, such as $\gamma$-amino-

() This is an Open-Access article distributed under the terms of the Creative Commons Attribution Non-Commercial License (http://creativecommons.org/licenses/by-nc/3.0) which permits unrestricted non-commercial use, distribution, and reproduction in any medium, provided the original work is properly cited. 
butyric acid (GABA) neurons, could account for the impairment of cortical inhibition after traumatic events. ${ }^{14)}$

Several studies have suggested that rTMS, with different treatment indices, such as intensity, stimulation location, and frequency, may be useful in the treatment of PTSD. The administration of low-frequency rTMS has been shown to be able to revert the lowered seizure threshold to normal, ${ }^{15,16)}$ and previous studies have reported that rTMS may alter brain function by modulating the release of neurotransmitters, the efficiency of synaptic transmission, and signal transduction pathways. ${ }^{17)}$

Given this background, we sought to assess the efficacy and tolerability of low-frequency rTMS in patients with PTSD over a 3-week period.

\section{METHODS}

Patients with PTSD were recruited from the PTSD clinic at The Catholic University of Korea, St. Mary's Hospital. The protocol was approved by the Institutional Review Board of The Catholic University of Korea (SCMC 070T076).

The traumatic events experienced by participants were non-military and included motor vehicle accidents $(\mathrm{n}=$ $13)$, domestic violence $(n=3)$, and physical assaults by strangers $(n=2)$. The mean time elapsed since the traumatic event was 3.3 years (standard deviation 1.4 years). Diagnosis according to the Diagnostic and Statistical Manual of Mental Disorders fourth edition, text revised version (DSM-IV-TR) criteria for PTSD was based on a consensus between two board-certified psychiatrists. ${ }^{18)}$
The Structured Clinical Interview, DSM-IV Axis I Disorders-Clinician Version (SCID) ${ }^{19)}$ was administered to all patients for lifetime comorbidities. Subjects with neurological illnesses and medico-surgical illnesses (e.g., seizure, estimated intelligence quotient less than 80 , and heart disease with a pacemaker) were excluded. Subjects were also excluded if they were pregnant or left-handed. The Clinician-Administered PTSD Scale (CAPS) ${ }^{20,21)}$ was administered to characterize PTSD symptoms quantitatively.

The 18 patients with PTSD were randomly assigned to the 1-Hz rTMS or sham condition according to a double-blind design. Sixteen patients with PTSD finally participated and completed the study. One patient withdrew consent prior to study initiation, and another dropped out after failing to keep the appointment schedule. The active rTMS group consisted of seven patients with PTSD, including three males and four females, with a mean age of $36.3 \pm 8.8$ years. The sham TMS group consisted of nine inpatients with PTSD (three males, six females; mean age: $32.8 \pm 6.9$ years). Demographic data are shown in Table 1 .

rTMS was performed using a TAMAS stimulator with a figure-of-8 coil (CR Tech, Daejon, Korea). On the initial treatment visit, we determined the motor threshold of the left abductor pollicis brevis muscle at rest. The resting motor threshold was defined as the minimum magnetic power needed to elicit contraction of the muscle, according to visual inspection, in five of 10 trials using single-pulse TMS administered to the contralateral primary motor cortex. The right prefrontal cortical stimulation site was defined as the location $5 \mathrm{~cm}$ anterior to and in a para-

Table 1. Demographic data for subjects

\begin{tabular}{|c|c|c|c|c|c|c|c|}
\hline Patient No. & Group & Age (year) & Sex & $\begin{array}{c}\text { Education } \\
\text { (year) }\end{array}$ & $\begin{array}{l}\text { Trauma/elapsed } \\
\text { time (year) }\end{array}$ & $\begin{array}{l}\text { CAPS score } \\
\text { at baseline }\end{array}$ & $\begin{array}{l}\text { Medication } \\
\text { (mg/day) }\end{array}$ \\
\hline 1 & Active & 45 & Male & 12 & TA 4.50 & 111 & Mirtazapine 30 \\
\hline 2 & Active & 28 & Male & 16 & DV 2.20 & 105 & Paroxetine 40 \\
\hline 3 & Active & 45 & Female & 14 & TA 3.10 & 111 & Sertraline 200 \\
\hline 4 & Active & 29 & Female & 12 & TA 4.80 & 99 & Paroxetine 40 \\
\hline 5 & Active & 25 & Female & 12 & TA 1.60 & 108 & Paroxetine 40 \\
\hline 6 & Active & 44 & Male & 9 & PA 5.20 & 103 & Mirtazapine 45 \\
\hline 7 & Active & 38 & Female & 16 & TA 1.40 & 106 & Mirtazapine 45 \\
\hline 8 & Sham & 26 & Female & 16 & DV 1.60 & 101 & Mirtazapine 45 \\
\hline 9 & Sham & 33 & Female & 16 & TA 4.90 & 107 & Mirtazapine 45 \\
\hline 10 & Sham & 33 & Male & 12 & TA 1.80 & 118 & Venlafaxine 112.5 \\
\hline 11 & Sham & 46 & Male & 9 & TA 4.50 & 99 & Sertraline 150 \\
\hline 12 & Sham & 33 & Female & 14 & TA 3.30 & 106 & Sertraline 200 \\
\hline 13 & Sham & 22 & Female & 12 & DV 2.40 & 111 & Paroxetine 40 \\
\hline 14 & Sham & 36 & Male & 12 & TA 5.10 & 102 & Paroxetine 40 \\
\hline 15 & Sham & 37 & Female & 12 & PA 2.50 & 104 & Paroxetine 30 \\
\hline 16 & Sham & 29 & Female & 12 & TA 3.60 & 92 & Mirtazapine 60 \\
\hline
\end{tabular}

CAPS, Clinician-Administered Posttraumatic Stress Disorder Scale; TA, traffic accident; DV, domestic violence; PA, physical assaults by stranger. 
sagittal plane from the site of maximal abductor pollicis brevis muscle stimulation, as in a previous study. ${ }^{22}$

The active rTMS group received 3 weeks of low-frequency rTMS trains at $1 \mathrm{~Hz}$ for 20 minutes per weekday (for a total of 15 days of treatment) at $100 \%$ of the motor threshold of the patient's resting motor threshold (total, 18,000 pulses) to the right prefrontal cortex. The sham group received an identical rTMS treatment, except that the lateral wing of the coil was raised $90^{\circ}$ off the head, with the edge of the medial wing of the coil still touching the scalp. While receiving active rTMS treatment or sham stimulation, patients were seated in a reclined, head-supported examination chair. rTMS was administered each day by a trained psychiatrist who purposefully had very limited verbal interaction with the subject. The rTMS administerer was blind to all subject information and blocked from communicating about subjects with raters. Prior to the study, the experimenter was trained to maintain a consistent and neutral attitude toward each practice to minimize biases.

Psychopharmacological and supportive psychotherapeutic approaches were maintained in both groups during the study period. Primary efficacy measures were the CAPS $^{20,21)}$ and its subscales, administered by an independent investigator blind to the stimulation conditions, at baseline and weeks 2, 4, and 8 .

Data from all participants were analyzed. For purposes of effectiveness, CAPS scores were analyzed using repeated-measures analysis of variance (ANOVA) with

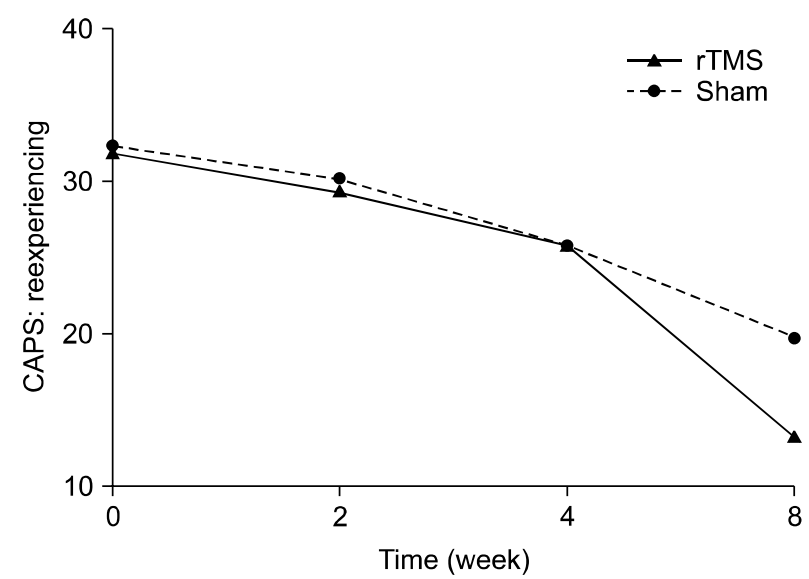

Fig. 1. Effects of $1-\mathrm{Hz}$ repetitive transcranial magnetic stimulation (rTMS) of $100 \%$ of motor threshold over right prefrontal cortex for 3 weeks comparing with sham stimulation in patients with posttraumatic stress disorder (re-experiencing subscale scores of Clinician-Administered Posttraumatic Stress Disorder Scale (CAPS)): repeated measures of analysis of variance; time effect ( $d f=1.707$, $F=146.0, p<0.001$ ), time by treatment group effect $(d f=1.707$, $F=7.47, p=0.004)$, treatment group effect $(d f=1, F=3.86, p=0.07)$.
CAPS score as the within-subject factor and assigned group as the between-subject factor. Interaction effects between time and treatment group were also considered. Post-hoc analysis was done to evaluate when the treatment effect actually happened using paired t-test. Descriptive statistics and the Monte-Carlo method were used to examine group differences where appropriate. All statistical significance calculations were two-tailed, and significance was set at $p<0.05$. All data analyses were conducted using Statistical Analysis System (SAS), version 9.1 (SAS Institute Inc., Cary, NC, USA).

\section{RESULTS}

The active rTMS and sham groups showed no statistically significant difference in baseline characteristics, including CAPS scores (Table 1). In terms of the time effect, we found significant improvements in all items on the CAPS. The interaction between time and treatment group revealed significant differences in the re-experiencing $(d f=1.707, F=7.47, p=0.004)$ and total $(d f=1.674, F=6.45$, $p=0.008)$ scores on the CAPS. The post-hoc analysis showed significant improvement in the re-experiencing $(p=0.004)$ and total $(p<0.001)$ scores on the CAPS of rTMS group from the time point of week 2 . The avoidance scores on the CAPS reflected a trend toward significance ( $d f=3, F=2.74, p=0.055$ ), but no significant difference in the CAPS hyperarousal scores was observed (Figs. 1-4).

No serious adverse effects of rTMS were reported by

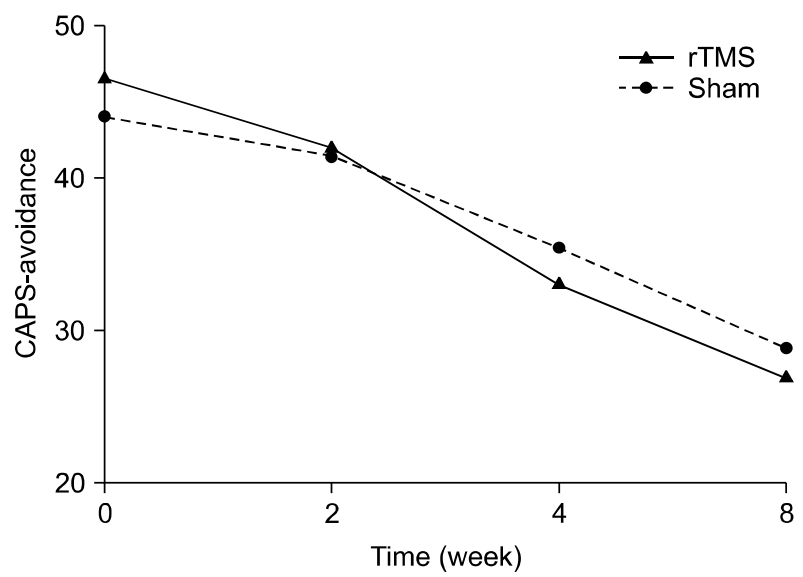

Fig. 2. Effects of $1-\mathrm{Hz}$ repetitive transcranial magnetic stimulation (rTMS) of $100 \%$ of motor threshold over right prefrontal cortex for 3 weeks comparing with sham stimulation in patients with posttraumatic stress disorder (avoidance subscale scores of Clinician-Administered Posttraumatic Stress Disorder Scale (CAPS)): repeated measures of analysis of variance; time effect ( $d f=3$, $F=120.3, p<0.001$ ), time by treatment group effect $(d f=3, F=2.74$, $p=0.055)$, treatment group effect ( $d f=1, F=0.04, p=0.846)$. 


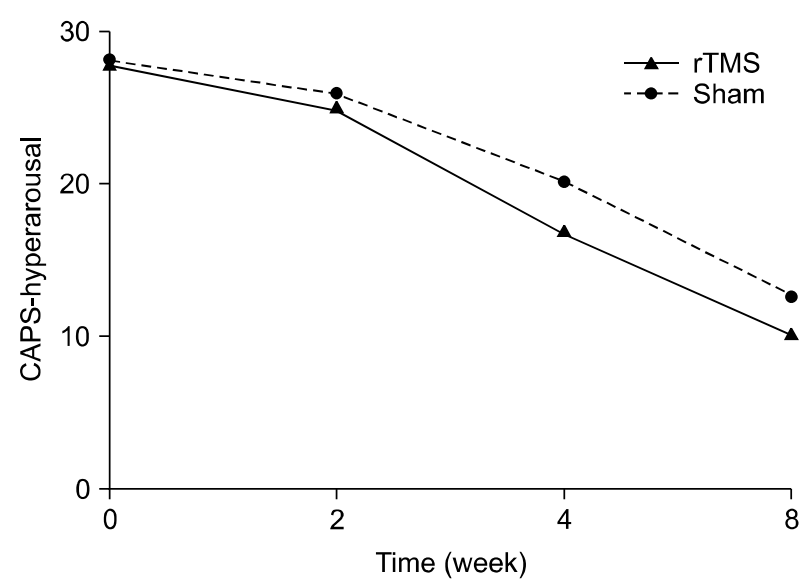

Fig. 3. Effects of $1-\mathrm{Hz}$ repetitive transcranial magnetic stimulation (rTMS) of $100 \%$ of motor threshold over right prefrontal cortex for 3 weeks comparing with sham stimulation in patients with posttraumatic stress disorder (hyperarousal subscale scores of Clinician-Administered Posttraumatic Stress Disorder Scale (CAPS)): repeated measures of analysis of variance; time effect (hypothesis $d f=3$, error $d f=12, F=64.73, p<0.001$ ), time by treatment group effect ( $d f=1.356, F=1.35, p=0.273$ ), treatment group effect $(d f=1, f=2.18, p=0.162)$.

the subjects during the trial. Mild adverse effects, such as headache $(n=3)$ and dizziness $(n=1)$, were reported during the real trial. Headache $(n=2)$, dizziness $(n=1)$, and difficulty in concentrating $(n=1)$ were reported during the sham trial.

\section{DISCUSSION}

In the present study, we found that 3 weeks of low-frequency rTMS on the right prefrontal cortex was associated with a greater therapeutic effect than was the sham control treatment among patients with PTSD. Moreover, the scores for the re-experiencing symptoms of PTSD showed significant improvement, although hyperarousal scores did not differ significantly between groups and the difference between the active rTMS and sham TMS groups with regard to avoidance was only marginally significant. This study suggests that rTMS treatment on the right prefrontal cortex may be a promising avenue for future research on the treatment of PTSD and that its differential effects may provide important information about specific PTSD symptom clusters.

Open trials ${ }^{23-25)}$ and a controlled study ${ }^{26)}$ have suggested that regimens of rTMS that differ in terms of such factors as frequency, stimulation intensity, and location of stimulation may have beneficial effects in the treatment of patients with PTSD. Grisaru et al. ${ }^{23)}$ performed low-frequency TMS with 15 repetitions of $0.3 \mathrm{~Hz}$ and observed a

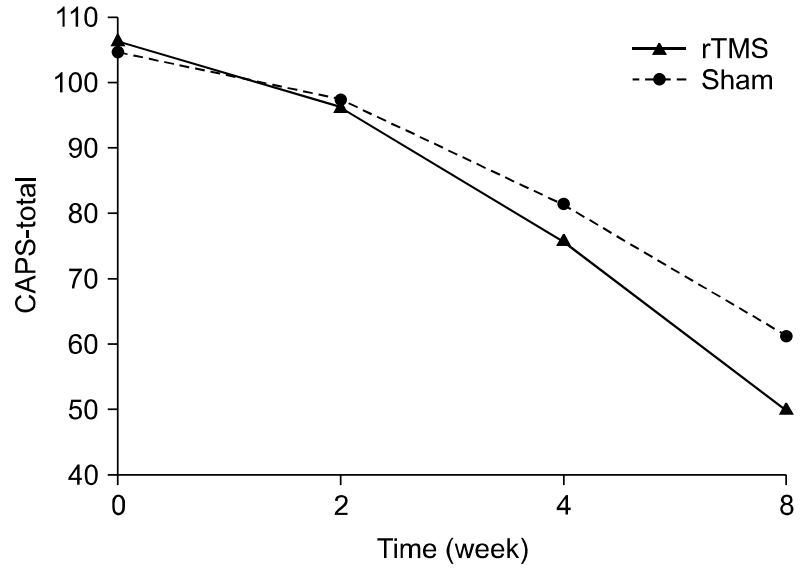

Fig. 4. Effects of $1-\mathrm{Hz}$ repetitive transcranial magnetic stimulation (rTMS) of $100 \%$ of motor threshold over right prefrontal cortex for 3 weeks comparing with sham stimulation in patients with posttraumatic stress disorder (total scores of Clinician-Administered Posttraumatic Stress Disorder Scale (CAPS)): repeated measures of analysis of variance; time effect ( $d f=1.674, F=387.67, p<0.001)$, time by treatment group effect ( $d f=1.674, F=6.45, p=0.008)$, treatment group effect ( $d f=1, f=2.36, p=0.147$ ).

temporary improvement in PTSD symptoms. McCann et $a l .{ }^{24)}$ used rTMS with a $1-\mathrm{Hz}$ low frequency and $80 \%$ motor threshold; PTSD core symptoms improved significantly, and positron emission tomography confirmed that the previously hyperactive metabolism in the right frontal lobe and the overall cerebrum decreased in two cases, suggesting the therapeutic effect of low-frequency rTMS for PTSD. A recent study ${ }^{25)}$ tried to find differences between low- and high-frequency stimulation in rTMS. In this study, six patients with comorbid PTSD and major depression were treated with $1 \mathrm{~Hz}$, and the other six patients were treated with $5 \mathrm{~Hz}$, with $90 \%$ motor threshold. The results showed only minimal improvement in PTSD symptoms, and the antidepressant effect of rTMS was not dependent on stimulation frequency. Using an $80 \%$ motor threshold, Cohen et al. ${ }^{26)}$ compared the effect of three conditions on individuals with PTSD: 1-Hz low frequency, 10-Hz high frequency, and sham treatment. They found that $10-\mathrm{Hz}$ high-frequency rTMS was associated with a greater therapeutic effect than was $1-\mathrm{Hz}$ low-frequency or sham stimulation. These results differed from those of previous trials $^{23-25)}$ and the present study, where low-frequency rTMS was effective in the treatment of PTSD. There are several possible reasons for these differences. Our results show no clear difference between the two groups until 2 weeks after 1-Hz rTMS; however, a significant difference was apparent after 4 and 8 weeks. It may be that the differences resulted from the more frequent stimulation, higher intensity, and longer duration of treatment and follow-up 
in our study than in Cohen et al.'s study, ${ }^{26)}$ although the effects of the natural course of PTSD and a small-sample bias may have been obscured in either study. As it appears that more stimuli of higher intensity had a more powerful antidepressant effect in previous studies, ${ }^{27)}$ the treatment protocol of Cohen's study, ${ }^{26)}$ with $1 \mathrm{~Hz}$ for 5 seconds per train and a 55-second intertrain interval for 20 minutes, may be somewhat weaker than that used in the present study. On other hand, an alternative explanation of the results of Cohen et al.'s study ${ }^{26)}$ was offered by Rossi et al. ${ }^{28)}$ Because high-frequency TMS over the frontal cortex could interfere with episodic, visual, ${ }^{29)}$ and auditory ${ }^{30)}$ memory, the authors suggested that rTMS in patients with PTSD could have beneficial effects on the classical manifestations of episodic memory dysfunction.

We also suggest that a gradual treatment effect could be linked to a delayed or progressive neuromodulatory effect associated with rTMS treatment. Thus, direct comparisons between low- and high-frequency rTMS and shortand long-term treatment periods would be useful.

The strengths of the present study include the 3-week treatment period and the higher intensity of the rTMS (100\% of motor threshold) compared with that in other studies. Most studies adopted short-term stimulation periods and relatively weak stimulation intensities, such as 1-2 weeks and $80-90 \%$, respectively. More intensive rTMS parameters might be more beneficial given the sustained effectiveness of rTMS up to 8 weeks in the present study. Indeed, enhanced outcomes have been suggested in association with different rTMS parameters, such as higher intensity and longer duration. ${ }^{15)}$ Recently, a similar study was conducted by Watts et al. ${ }^{31)}$ Their study also showed clinically significant improvement in PTSD symptoms with $1-\mathrm{Hz}$ rTMS over the right dorsolateral prefrontal cortex, but the effectiveness showed some degradation when evaluated at 2 months post-treatment. There was also no difference in the effectiveness of rTMS across the three clusters of core PTSD symptoms. However, that study also used a relatively low intensity and few total pulses compared with our study.

It remains unclear as to which areas of the brain are responsible for the manifestation of and improvement in PTSD symptoms. With the exception of one small study, ${ }^{25)}$ most studies have stimulated the right prefrontal cortex as the treatment of PTSD and, as evidenced by some imaging data ${ }^{13,32)}$ and animal studies, ${ }^{33)}$ the right prefrontal cortex may be more closely associated with PTSD than is the left side. In a recent study, ${ }^{34)}$ patients with
PTSD showed marked increases in glutamatergic intracortical facilitation in the right hemisphere; they also showed right-sided impairment of short latency afferent inhibition, reflecting GABA-mediated modulation. These findings may be associated with the lateralization of the hemispheres in PTSD. Additionally, the study conducted by Pallanti et al. ${ }^{35)}$ compared the effect of unilateral stimulation with rTMS and sequential bilateral stimulation in treatment-resistant depressed patients. This study demonstrated that low-frequency rTMS over the unilateral right dorsolateral prefrontal cortex showed higher efficacy than did bilateral stimulation. The results also suggested that low-frequency unilateral stimulation was more attractive than was bilateral stimulation in terms of safety, tolerability, and cost-effectiveness.

Interestingly, in terms of subscore comparisons, only the re-experiencing score was improved significantly in the active rTMS group versus the sham group, which is consistent with a previous study. ${ }^{26)}$ Additionally, the typical antidepressant pharmacotherapy for PTSD would be expected to affect the depressive symptoms of PTSD rather than the core symptoms, ${ }^{36)}$ suggesting that a differential effect of rTMS on the specific core PTSD symptom cluster may occur.

The present study has several limitations. The most important limitation is the small number of subjects, corresponding to power of $45 \%$ with an effect size of 0.8 at a two-tailed significance level of less than 0.05 . Second, as our observation period was somewhat long, we cannot completely exclude progressive changes in relation to the natural course of the disease, and the placebo effect of rTMS has also been debated. ${ }^{37,38)}$ Third, patients continued to receive medications. Fourth, we did not assess comorbidities, such as depression or other anxiety disorders. Finally, we used only low-frequency rTMS with no comparison arm.

In conclusion, despite several methodological weakness, the present study showed that low-frequency rTMS on the right prefrontal cortex for 3 weeks was associated with therapeutic effects compared with sham treatment among patients with PTSD and also demonstrated differential effects on the core PTSD symptom cluster.

\section{Acknowledgments}

This work was supported by grant of Korean Research Foundation (2012-0006579) and Seoul R\&BD Program (SS110008). 


\section{REFERENCES}

1. Post RM, Kimbrell TA, McCann UD, Dunn RT, Osuch EA, Speer AM, et al. Repetitive transcranial magnetic stimulation as a neuropsychiatric tool: present status and future potential. J ECT 1999;15:39-59.

2. Ben-Shachar D, Belmaker RH, Grisaru N, Klein E. Transcranial magnetic stimulation induces alterations in brain monoamines. J Neural Transm 1997;104:191-197.

3. Weiss SR, Li XL, Rosen JB, Li H, Heynen T, Post RM. Quenching: inhibition of development and expression of amygdala kindled seizures with low frequency stimulation. Neuroreport 1995;6:2171-2176.

4. George MS, Wassermann EM, Williams WA, Steppel J, Pascual-Leone A, Basser P, et al. Changes in mood and hormone levels after rapid-rate transcranial magnetic stimulation (rTMS) of the prefrontal cortex. J Neuropsychiatry Clin Neurosci 1996;8:172-180.

5. George MS, Nahas Z, Lisanby SH, Schlaepfer T, Kozel FA, Greenberg BD. Transcranial magnetic stimulation. Neurosurg Clin N Am 2003;14:283-301.

6. Greenberg BD, George MS, Martin JD, Benjamin J, Schlaepfer TE, Altemus M, et al. Effect of prefrontal repetitive transcranial magnetic stimulation in obsessivecompulsive disorder: a preliminary study. Am J Psychiatry 1997; 154:867-869.

7. Ingham RJ, Fox PT, Ingham JC, Collins J, Pridgen S. TMS in developmental stutturing and Tourette's syndrome. In: George MS, Belmaker RH, editors. Transcranial magnetic stimulation in neuropsychiatry. Washington, DC:American Psychiatric Press;2000. p.223-236.

8. Daskalakis ZJ, Christensen BK, Chen R, Fitzgerald PB, Zipursky RB, Kapur S. Evidence for impaired cortical inhibition in schizophrenia using transcranial magnetic stimulation. Arch Gen Psychiatry 2002;59:347-354.

9. Lee SH, Kim W, Chung YC, Jung KH, Bahk WM, Jun TY, et al. A double blind study showing that two weeks of daily repetitive TMS over the left or right temporoparietal cortex reduces symptoms in patients with schizophrenia who are having treatment-refractory auditory hallucinations. Neurosci Lett 2005;376:177-181.

10. Bremner JD, Innis RB, Ng CK, Staib LH, Salomon RM, Bronen RA, et al. Positron emission tomography measurement of cerebral metabolic correlates of yohimbine administration in combat-related posttraumatic stress disorder. Arch Gen Psychiatry 1997;54:246-254.

11. Rauch SL, van der Kolk BA, Fisler RE, Alpert NM, Orr SP, Savage CR, et al. A symptom provocation study of posttraumatic stress disorder using positron emission tomography and script-driven imagery. Arch Gen Psychiatry 1996;53:380-387.

12. Shin LM, McNally RJ, Kosslyn SM, Thompson WL, Rauch $\mathrm{SL}$, Alpert NM, et al. A positron emission tomographic study of symptom provocation in PTSD. Ann N Y Acad Sci 1997;821:521-523.

13. Shin LM, Kosslyn SM, McNally RJ, Alpert NM, Thompson $\mathrm{WL}$, Rauch SL, et al. Visual imagery and perception in posttraumatic stress disorder. A positron emission tomographic investigation. Arch Gen Psychiatry 1997;54:233241.

14. Centonze D, Palmieri MG, Boffa L, Pierantozzi M, Stanzione $\mathrm{P}$, Brusa L, et al. Cortical hyperexcitability in post-traumatic stress disorder secondary to minor accidental head trauma: a neurophysiologic study. J Psychiatry Neurosci 2005;30:127-132.
15. George MS, Lisanby SH, Sackeim HA. Transcranial magnetic stimulation: applications in neuropsychiatry. Arch Gen Psychiatry 1999;56:300-311.

16. McEwen BS. The neurobiology and neuroendocrinology of stress. Implications for post-traumatic stress disorder from a basic science perspective. Psychiatr Clin North Am 2002; 25:469-494.

17. Post A, Keck ME. Transcranial magnetic stimulation as a therapeutic tool in psychiatry: what do we know about the neurobiological mechanisms? J Psychiatr Res 2001;35:193215.

18. American Psychiatric Association. Diagnostic and statistical manual of mental disorder, 4th ed. Washington, DC:American Psychiatric Press; 1994.

19. First MB, Spitzer RL, Gibbon M, Williams JBW. Structured clinical interview for DSM-IV-Clinician version (SCID-CV). Washington, DC:American Psychiatric Press;1997.

20. Blake DD, Weathers FW, Nagy LM, Kaloupek DG, Gusman FD, Charney DS, et al. The development of a ClinicianAdministered PTSD Scale. J Trauma Stress 1995;8:75-90.

21. Weathers FW, Litz BT. Psychometric properties of the Clinician-Administered PTSD Scale, CAPS-1. PTSD Res $Q$ 1994;5:2-6.

22. Pascual-Leone A, Catalá MD, Pascual-Leone Pascual A. Lateralized effect of rapid-rate transcranial magnetic stimulation of the prefrontal cortex on mood. Neurology 1996;46: 499-502.

23. Grisaru N, Amir M, Cohen H, Kaplan Z. Effect of transcranial magnetic stimulation in posttraumatic stress disorder: a preliminary study. Biol Psychiatry 1998;44:52-55.

24. McCann UD, Kimbrell TA, Morgan CM, Anderson T, Geraci M, Benson BE, et al. Repetitive transcranial magnetic stimulation for posttraumatic stress disorder. Arch Gen Psychiatry 1998;55:276-279.

25. Rosenberg PB, Mehndiratta RB, Mehndiratta YP, Wamer A, Rosse RB, Balish M. Repetitive transcranial magnetic stimulation treatment of comorbid posttraumatic stress disorder and major depression. J Neuropsychiatry Clin Neurosci 2002;14:270-276.

26. Cohen H, Kaplan Z, Kotler M, Kouperman I, Moisa R, Grisaru N. Repetitive transcranial magnetic stimulation of the right dorsolateral prefrontal cortex in posttraumatic stress disorder: a double-blind, placebo-controlled study. Am J Psychiatry 2004;161:515-524.

27. Chae JH, Nahas Z, Li X, George MS. Transcranial magnetic stimulation in psychiatry: research and therapeutic application. Int Rev Psychiatry 2001;13:18-23.

28. Rossi S, Cappa SF, Ulivelli M, De Capua A, Bartalini S, Rossini PM. rTMS for PTSD: induced merciful oblivion or elimination of abnormal hypermnesia? Behav Neurol 2006; 17:195-199.

29. Rossi S, Cappa SF, Babiloni C, Pasqualetti P, Miniussi C, Carducci F, et al. Prefrontal [correction of Prefontal] cortex in long-term memory: an "interference" approach using magnetic stimulation. Nat Neurosci 2001;4:948-952.

30. Sandrini M, Cappa SF, Rossi S, Rossini PM, Miniussi C. The role of prefrontal cortex in verbal episodic memory: rTMS evidence. J Cogn Neurosci 2003;15:855-861.

31. Watts BV, Landon B, Groft A, Young-Xu Y. A sham controlled study of repetitive transcranial magnetic stimulation for posttraumatic stress disorder. Brain Stimul 2012;5:3843

32. Semple WE, Goyer P, McCormick R, Morris E, Compton B, Muswick G, et al. Preliminary report: brain blood flow using PET in patients with posttraumatic stress disorder and 
substance-abuse histories. Biol Psychiatry 1993;34:115-118.

33. Morgan MA, LeDoux JE. Differential contribution of dorsal and ventral medial prefrontal cortex to the acquisition and extinction of conditioned fear in rats. Behav Neurosci 1995; 109:681-688.

34. Rossi S, De Capua A, Tavanti M, Calossi S, Polizzotto NR, Mantovani A, et al. Dysfunctions of cortical excitability in drug-naïve posttraumatic stress disorder patients. Biol Psychiatry 2009;66:54-61.

35. Pallanti S, Bernardi S, Di Rollo A, Antonini S, Quercioli L. Unilateral low frequency versus sequential bilateral repetitive transcranial magnetic stimulation: is simpler better for treatment of resistant depression? Neuroscience 2010;167:323-328.

36. Randall PK, Bremner JD, Krystal JH, Nagy LM, Heninger
GR, Nicolaou AL, et al. Effects of the benzodiazepine antagonist flumazenil in PTSD. Biol Psychiatry 1995;38: 319-324.

37. George MS, Wassermann EM, Kimbrell TA, Little JT, Williams WE, Danielson AL, et al. Mood improvement following daily left prefrontal repetitive transcranial magnetic stimulation in patients with depression: a placebocontrolled crossover trial. Am J Psychiatry 1997;154:17521756.

38. Klein E, Kreinin I, Chistyakov A, Koren D, Mecz L, Marmur S, et al. Therapeutic efficacy of right prefrontal slow repetitive transcranial magnetic stimulation in major depression: a double-blind controlled study. Arch Gen Psychiatry 1999;56:315-320. 